\title{
Generic Regular Decompositions for Parametric Polynomial Systems
}

\author{
Zhenghong Chen* Xiaoxian Tang Bican Xia \\ LMAM \& School of Mathematical Sciences \\ Peking University, Beijing 100871, China \\ chenzhenghong@pku.edu.cn, tangxiaoxian@pku.edu.cn, xbc@math.pku.edu.cn
}

\begin{abstract}
This paper presents a generalization of our earlier work in 19. In this paper, the two concepts, generic regular decomposition (GRD) and regular-decomposition-unstable (RDU) variety introduced in [19] for generic zero-dimensional systems, are extended to the case where the parametric systems are not necessarily zero-dimensional. An algorithm is provided to compute GRDs and the associated RDU varieties of parametric systems simultaneously on the basis of the algorithm for generic zero-dimensional systems proposed in [19]. Then the solutions of any parametric system can be represented by the solutions of finitely many regular systems and the decomposition is stable at any parameter value in the complement of the associated RDU variety of the parameter space. The related definitions and the results presented in 19 are also generalized and a further discussion on RDU varieties is given from an experimental point of view. The new algorithm has been implemented on the basis of DISCOVERER 28] with Maple 16 and experimented with a number of benchmarks from the literature.
\end{abstract}

Keywords: parametric polynomial system, regular-decomposition-unstable variety, generic regular decomposition

\section{Introduction}

As is well known, solving parametric polynomial system plays a key role in many application fields such as automated geometry theorem deduction, stability analysis of dynamical systems, robotics and so on. To solve a parametric system symbolically, a basic idea is to transform the system into new systems with special structures or properties so that the solutions of the original system can be handled via studying the solutions of the new systems, which is relatively easy. Remarkable examples of such methods are the algorithms for computing comprehensive Gröbner systems (CGS) and comprehensive Gröbner bases (CGB) [26, 12, 15, 16, 17, 18. The methods based on triangular decompositions are another kind of such examples [1, 4, 7, 11, 13, 20, 22, 27, 29, 31, 32,

Since Wu's work 27, lots of well-known methods based on triangular decompositions have been proposed. An essential concept, "regular chain" (or "normal chain"), and algorithms for computing regular chain decomposition have been introduced by Kalkbrener 11 and Yang and Zhang 32 independently. For parametric systems, Gao and Chou proposed a method in 7 for identifying all parametric values for which a given system has solutions and giving the solutions by $p$-chains without a partition of the parameter space. Wang gave an efficient algorithm for computing regular system decomposition [22, 23, 25], which is a generalization of regular chain decomposition. The concept of comprehensive triangular decomposition (CTD) introduced by Chen et al. in [4] is an analogue of the CGS for solving parametric polynomial systems.

Two new concepts, generic regular decomposition and regular-decomposition-unstable (RDU) variety for generic zero-dimensional systems, are introduced in [19] and an algorithm is proposed for computing a generic regular decomposition and the associated RDU variety of a given generic

*Corresponding author Email:chenzhenghong@pku.edu.cn 
zero-dimensional system simultaneously. The solutions of the given system can be expressed by finitely many zero-dimensional regular chains if the parameter value is not on the RDU variety. The so-called weakly relatively simplicial decomposition (WRSD) plays a crucial role in the algorithm, which is based on the theories of subresultants.

In this paper, the concepts, generic regular decomposition (GRD) and regular-decompositionunstable (RDU) variety, introduced in [19] for generic zero-dimensional systems are extended to the case where the parametric systems are not necessarily zero-dimensional. An algorithm is provided to compute GRDs and the associated RDU varieties of parametric systems simultaneously on the base of the algorithm for generic zero-dimensional systems proposed in [19. Then the solutions of any parametric system can be represented by the solutions of finitely many regular systems and the decomposition is stable at any parameter value in the complement of the associated RDU variety of the parameter space. The new algorithm has been implemented on the base of DISCOVERER 28] with Maple 16 and experimented with a number of benchmarks from the literature 4, 9, 12, 15, 16. Empirical results are also presented to show the good performance of the algorithm. In other words, this paper presents a generalization of our earlier work in 19 .

First of all, we need to introduce the idea proposed in 19] briefly. For a given generic zero-dimensional system $\mathbf{P}$ with $n$ variables and $d$ parameters, we considered the parameters as "constants" and proposed Algorithm RDUForZD for computing a so-called generic regular decomposition $\mathbb{T}$ of $\mathbf{P}$ in $\overline{K(U)}^{n}$ such that $\mathrm{V}_{\overline{K(U)}}(\mathbf{P})=\cup_{\mathbf{T} \in \mathbb{T}} \mathrm{V}_{\overline{K(U)}}\left(\mathbf{T} \backslash \mathrm{I}_{\mathbf{T}}\right)$, where $\mathbb{T}$ is a set of regular chains. At the same time, the algorithm would obtain a parametric polynomial such that the regular decomposition was stable at any parametric point outside the variety (called RDU variety) generated by the parametric polynomial. Roughly speaking, "stable at a parametric point" means that the regular decomposition remains after we substitute the point for the parameters in $\mathbf{P}$ and $\mathbb{T}$ (see Definition 5). As a result, the original generic zero-dimensional system is "solved" except for the case where parameters are on the RDU variety. That is why the decomposition is called generic regular decomposition.

Now we would like to show some new ideas of this paper. If the given system is not generic zero-dimensional, to obtain a decomposition with similar properties as in the zero-dimensional case, we choose to express the solutions of the system by finitely many regular systems 23$]$ instead of regular chains. So we need to generalize the concept "generic regular (chain) decomposition" introduced in 19 into "generic regular (system) decomposition" (see Definitions 2, 3 and 5). For solving a positive dimensional system, a natural idea is to view some variables as parameters and call recursively the algorithm for generic zero-dimensional systems proposed in [19. However, to prove the correctness of this procedure, we need to study the properties of characteristic sets under specifications carefully (see Lemma 2 and Corollary 21). Besides, it is worth to notice that we have two different interpretations for the results computed by Algorithm ZDToRC and both of them play a key role in the proof of the correctness (see Lemma 3). Finally, we give an algorithm which, for any parametric system $\mathbf{P}$, computes a finite set $\mathbb{T} \mathbb{H}$ of regular systems in $K[U][X]$ and a polynomial $B \in K[U]$, such that

1. $\mathrm{V}_{\overline{K(U)}}(\mathbf{P})=\cup_{[\mathbf{T}, H] \in \mathbb{H}} \mathrm{V}_{\overline{K(U)}}(\mathbf{T} \backslash H)$; and

2. for any $a \in \bar{K}^{d} \backslash \mathrm{V}^{U}(B), \mathrm{V}(\mathbf{P}(a))=\cup_{[\mathbf{T}, H] \in \mathbb{T H}} \mathrm{V}(\mathbf{T}(a) \backslash H(a))$ and $[\mathbf{T}, H]$ specializes well at $a$ for any $[\mathbf{T}, H] \in \mathbb{T} H$.

Please see Algorithm 4 in this paper for more details. What's more, for different orderings of variables, the efficiency of the algorithm can be different and the RDU varieties can be totally different.

At the end of this section, it is worth to point out that the algorithm provided in this paper has a different feature compared to some existing algorithms. The algorithm for computing regular system decomposition proposed in 22, 23. uses the so-called variable elimination, which computes a main branch at first and then gets the other branches one by one. An incremental algorithm, introduced in [4, 8, for computing regular chain decomposition, computes a regular chain decomposition for some polynomials in the given system at first and then intersects the other polynomials with the regular chains one by one. The algorithm proposed in this paper makes use of a hierarchical strategy. From an experimental point of view, different strategies are suitable for different benchmarks (see Section 4). 
The paper is organized as follows. Section 2 provides basic definitions and concepts that are needed to understand the main algorithm. Section 3 contains the main algorithm, namely Algorithm 4, and some relative subalgorithms. Also we review the description of algorithms in our former article. Besides, proofs for these algorithms are presented in this section. Some illustrative examples, the empirical data and comparison with previous work along with several implementation details are presented in Section 4. Section 5]concludes the paper with a discussion on our future work along this direction.

\section{Preliminaries}

The following paragraphs give a brief outline of the vocabulary and tools we will be using throughout the paper. All concepts without precise definitions can be found in [5, 27, 31]. $\mathbb{R}$ and $\mathbb{C}$ stand for the feild of real numbers and the field of complex numbers, respectively.

Suppose $\left\{u_{1}, \ldots, u_{d}, x_{1}, \ldots, x_{n}\right\}$ is a set of indeterminates with a given order $u_{1} \prec \ldots \prec u_{d} \prec$ $x_{1} \prec \ldots \prec x_{n}$ where $\left\{u_{1}, \ldots, u_{d}\right\}$ and $\left\{x_{1}, \ldots, x_{n}\right\}$ are the sets of parameters and variables, respectively. Let $U=\left\{u_{1}, \ldots, u_{d}\right\}$ and $X=\left\{x_{1}, \ldots, x_{n}\right\}$. Suppose $K$ is a field and $\bar{K}$ denotes its algebraic closure. Let $K[U]$ be the ring of polynomials in $U$ with coefficients in $K$ and $K(U)$ be the rational function field. A non-empty finite subset $\mathbf{P}$ of $K[U][X]$ is said to be a polynomial system or system. If $\mathbf{P} \subset K[U][X] \backslash K[X]$, it is a parametric polynomial system or parametric system. If $\mathbf{P} \subset K[X]$, it is a constant polynomial system or constant system.

For a non-empty finite subset $\mathbf{P} \subset K[U][X](\bar{K}[X]),\langle\mathbf{P}\rangle_{K[U][X]}\left(\langle\mathbf{P}\rangle_{\bar{K}[X]}\right)$ denotes the ideal generated by $\mathbf{P}$ in $K[U][X](\bar{K}[X])$ and $\sqrt{\langle\mathbf{P}\rangle_{K[U][X]}}\left(\sqrt{\langle\mathbf{P}\rangle_{\bar{K}[X]}}\right)$ denotes the radical ideal of $\langle\mathbf{P}\rangle_{K[U][X]}\left(\langle\mathbf{P}\rangle_{\bar{K}[X]}\right)$. For any $F$ in $K[U][X] \backslash\{0\}(\bar{K}[X] \backslash\{0\})$ and for any $x \in X$, if $x$ appears in $F, F$ can be regarded as a univariate polynomial in $x$, namely $F=C_{0} x^{m}+C_{1} x^{m-1}+\ldots+C_{m}$ where $C_{0}, C_{1}, \ldots, C_{m}$ are polynomials in $K[U][X \backslash\{x\}](\bar{K}[X \backslash\{x\}])$ and $C_{0} \neq 0$. Then $m$ is the leading degree of $F$ w.r.t. $x$ and is denoted by $\operatorname{deg}(F, x)$. Note that if $x$ does not appear in $F$, $\operatorname{deg}(F, x)=0$. If there exists $p(1 \leq p \leq n) \operatorname{such}$ that $\operatorname{deg}\left(F, x_{p}\right)>0$ and for every $i(p<i \leq n)$, $\operatorname{deg}\left(F, x_{i}\right)=0$, then the class of $F$ is $p$. If $\operatorname{deg}\left(F, x_{i}\right)=0$ for every $i(1 \leq i \leq n)$, then the class of $F$ is 0 . The class of $F$ in $K[U][X] \backslash\{0\}(\bar{K}[X] \backslash\{0\})$ is denoted by $\operatorname{cls}_{F}$. If $\operatorname{cls}_{F}>0, x_{\operatorname{cls}_{F}}$ is the main variable of $F$ and is denoted by $\operatorname{mvar}(F)$. Assume that $F=C_{0} x_{p}^{m}+C_{1} x_{p}^{m-1}+\ldots+C_{m}$ where $p=\operatorname{cls}_{F}>0$ and $C_{0} \neq 0$, then $C_{0}$, denoted by $\mathrm{I}_{F}$, is the initial of $F$ and $x_{p}^{m}$, denoted by $\operatorname{rank}(F)$, is the $\operatorname{rank}$ of $F$.

For any $\mathbf{P} \subset K[U][X], \mathrm{V}_{\bar{K}}(\mathbf{P})$ denotes the set $\left\{\left(a_{1}, \ldots, a_{d+n}\right) \in \bar{K}^{d+n} \mid P\left(a_{1}, \ldots, a_{d+n}\right)=\right.$ $0, \forall P \in \mathbf{P}\}$. For any $\mathbf{B} \subset K[U], \mathrm{V}^{U}(\mathbf{B})$ denotes the set $\left\{\left(a_{1}, \ldots, a_{d}\right) \in \bar{K}^{d} \mid B\left(a_{1}, \ldots, a_{d}\right)=\right.$ $0, \forall B \in \mathbf{B}\}$. And $\mathrm{V}_{\overline{K(U)}}(\mathbf{P})$ denotes the set $\left\{\left(a_{1}, \ldots, a_{n}\right) \in \overline{K(U)}^{n} \mid P\left(U, a_{1}, \ldots, a_{n}\right)=0, \forall P \in\right.$ $\mathbf{P}\}$. For any $F \in K[U][X]$, the coefficients $B_{1}, \ldots, B_{t}$ of $F$ in $X$ are polynomials in $K[U]$, then $\mathrm{V}^{U}(F)$ denotes $\mathrm{V}^{U}\left(\left\{B_{1}, \ldots, B_{t}\right\}\right)$. Note that for two finite subsets $\mathbf{P}$ and $\mathbf{H}$ of $K[U][X]$, $\mathrm{V}_{\overline{K(U)}}(\mathbf{P} \backslash \mathbf{H})$ denotes the set $\mathrm{V}_{\overline{K(U)}}(\mathbf{P}) \backslash \mathrm{V}_{\overline{K(U)}}(\mathbf{H})$. Similarly, we can have $\mathrm{V}(\mathbf{P} \backslash \mathbf{H}), \mathrm{V}_{\bar{K}}(\mathbf{P} \backslash \mathbf{H})$ and $\mathrm{V}^{U}(\mathbf{P} \backslash \mathbf{H}) \operatorname{dim}(\mathcal{V})$ denotes the dimension of an affine variety $\mathcal{V}$.

Triangular Set. A non-empty finite set $\mathbf{T}=\left\{T_{1}, T_{2} \ldots, T_{r}\right\}$ of polynomials in $K[U][X]$ $(\bar{K}[X])$ is a triangular set in $K[U][X](\bar{K}[X])$ if $0<\operatorname{cls}_{T_{1}}<\operatorname{cls}_{T_{2}}<\ldots<\operatorname{cls}_{T_{r}}$. For a triangular set $\mathbf{T}$ in $K[U][X](\bar{K}[X]), \mathrm{I}_{\mathbf{T}}, \operatorname{mvar}(\mathbf{T})$ and $\operatorname{rank}(\mathbf{T})$ denote $\Pi_{T \in \mathbf{T}} \mathrm{I}_{T},\{\operatorname{mvar}(T) \mid T \in \mathbf{T}\}$ and $\{\operatorname{rank}(T) \mid T \in \mathbf{T}\}$, respectively. The saturated ideal of a triangular set $\mathbf{T}$ in $K[U][X]$ is defined as the set $\left\{F \in K[U][X] \mid \mathrm{I}_{\mathbf{T}}{ }^{s} F \in\langle\mathbf{T}\rangle_{K[U][X]}\right.$ for some positive integer $\left.s\right\}$ and is denoted by $\operatorname{sat}(\mathbf{T})_{K[U][X]}$. Similarly, the saturated ideal of a triangular set $\mathbf{T}$ in $\bar{K}[X]$ is defined as the set $\left\{F \in \bar{K}[X] \mid \mathrm{I}_{\mathbf{T}}{ }^{s} F \in\langle\mathbf{T}\rangle_{\bar{K}[X]}\right.$ for some positive integer $\left.s\right\}$ and is denoted by $\operatorname{sat}(\mathbf{T})_{\bar{K}[X]}$. Suppose $F \in K[U][X](\bar{K}[X])$ and $\mathbf{T}$ is a triangular set in $K[U][X](\bar{K}[X])$, then $F$ is reduced w.r.t. $\quad \mathbf{T}$ if $\operatorname{deg}\left(F, \operatorname{mvar}\left(T_{i}\right)\right)<\operatorname{deg}\left(T_{i}, \operatorname{mvar}\left(T_{i}\right)\right)$ for every $i(1 \leq i \leq r)$. A triangular set $\mathbf{T}=\left\{T_{1}, T_{2} \ldots, T_{r}\right\}$ in $K[U][X](\bar{K}[X])$ is a non-contradictory ascending chain in $K[U][X]$ $(\bar{K}[X])$ if $T_{i}$ is reduced w.r.t. $\left\{T_{1}, \ldots, T_{i-1}\right\}$ for every $i(2 \leq i \leq r)$. A single-element set $\{F\} \subset K[U](\{F\} \subset \bar{K})$ is a contradictory ascending chain in $K[U][X](\bar{K}[X])$ if $F \neq 0$. Remark that an ascending chain is either a non-contradictory ascending chain or a contradictory ascending chain. 
Successive Pseudo Remainder. For two polynomials $F$ and $P$ in $K[U][X](\bar{K}[X])$ and a variable $x \in X$, the pseudo remainder of $F$ pseudo-divided by $P$ w.r.t. $x$ is denoted by $\operatorname{prem}(F, P, x)$. Particularly, $\operatorname{prem}(F, P, \operatorname{mvar}(P))$ is denoted by $\operatorname{prem}(F, P)$. For a polynomial $F \in K[U][X](\bar{K}[X])$ and a triangular set $\mathbf{T}=\left\{T_{1}, \ldots, T_{r}\right\}$ in $K[U][X](\bar{K}[X])$, the successive pseudo remainder 32 of $F$ w.r.t. $\mathbf{T}$ is denoted by $\operatorname{prem}(F, \mathbf{T})$, namely

$$
\operatorname{prem}(F, \mathbf{T})=\operatorname{prem}\left(\ldots \operatorname{prem}\left(\operatorname{prem}\left(F, T_{r}\right), T_{r-1}\right), \ldots, T_{1}\right) .
$$

For a finite set $\mathbf{P} \subset K[U][X](\bar{K}[X]), \operatorname{prem}(\mathbf{P}, \mathbf{T})$ denotes the set $\{\operatorname{prem}(F, \mathbf{T}) \mid F \in \mathbf{P}\}$.

Successive Resultant. For two polynomials $F$ and $P$ in $K[U][X](\bar{K}[X])$ and a variable $x \in$ $X$, the resultant 32 of $F$ and $P$ w.r.t. $x$ is denoted by $\operatorname{res}(F, P, x)$. Particularly, $\operatorname{res}(F, P, \operatorname{mvar}(P))$ is denoted by $\operatorname{res}(F, P)$. For a polynomial $F \in K[U][X](\bar{K}[X])$ and a triangular set $\mathbf{T}=$ $\left\{T_{1}, \ldots, T_{r}\right\}$ in $K[U][X](\bar{K}[X])$, the successive resultant $[32]$ of $F$ w.r.t. $\mathbf{T}$ is denoted by $\operatorname{res}(F, \mathbf{T})$, namely

$$
\operatorname{res}(F, \mathbf{T})=\operatorname{res}\left(\ldots \operatorname{res}\left(\operatorname{res}\left(F, T_{r}\right), T_{r-1}\right), \ldots, T_{1}\right) .
$$

Regular Chain. A triangular set $\mathbf{T}=\left\{T_{1}, \ldots, T_{r}\right\}$ in $K[U][X](\bar{K}[X])$ is said to be a regular chain in $K[U][X](\bar{K}[X])$, if $\mathrm{I}_{T_{1}} \neq 0$ and for each $i(1<i \leq r)$, $\operatorname{res}\left(\mathrm{I}_{T_{i}},\left\{T_{i-1}, \ldots, T_{1}\right\}\right) \neq 0$. If $\mathbf{T}$ is a regular chain in $K[U][X](\bar{K}[X])$ and $\operatorname{mvar}(\mathbf{T})=X, \mathbf{T}$ is a zero-dimensional regular chain.

Regular System 1 . [4 Let $\mathbf{T} \subset K[U][X](\bar{K}[X])$ be a regular chain and $H \in K[U][X](\bar{K}[X])$. If $\operatorname{res}(H, \mathbf{T}) \neq 0$, then $[\mathbf{T}, H]$ is said to be a regular system in $K[U][X](\bar{K}[X])$.

Proposition 1. 4 If $[\mathbf{T}, H]$ is a regular system in $K[U][X]$, then $\mathrm{V}_{\overline{K(U)}}(\mathbf{T} \backslash H) \neq \emptyset$.

Assignment Homomorphism. For each $a=\left(a_{1}, \ldots, a_{d}\right) \in \bar{K}^{d}, \phi_{a}: K[U][X] \longrightarrow \bar{K}[X]$ is a homomorphism such that $\phi_{a}(F)=F(a, X)$ for all $F \in K[U][X]$ and we denote $\phi_{a}(F)$ by $F(a)$. For a non-empty finite set $\mathbf{P} \subset K[U][X], \mathbf{P}(a)$ denotes the set $\{F(a) \mid F \in \mathbf{P}\}$ and remark that $\mathbf{P}(a)=\emptyset$ if $\mathbf{P}=\emptyset$.

Characteristic Set And Wu's Method. An ascending chain $\mathbf{C}$ in $K[U][X]$ is a characteristic set of $\mathbf{P}$ in $K[U][X]$ if $\mathbf{C} \subset\langle\mathbf{P}\rangle_{K[U][X]}$ and $\operatorname{prem}(\mathbf{P}, \mathbf{C})=\{0\}$. Theorem 1 below is the so called well-ordering principle.

Theorem 1. 27 There exists an algorithm which, for an input non-empty finite subset $\mathbf{P} \subset$ $K[U][X]$, outputs either a contradictory ascending chain meaning that $\mathrm{V}_{\overline{K(U)}}(\mathbf{P})=\emptyset$, or a (noncontradictory) characteristic set $\mathbf{C}=\left\{C_{1}, \ldots, C_{t}\right\}$ such that

$$
\mathrm{V}_{\overline{K(U)}}(\mathbf{P})=\mathrm{V}_{\overline{K(U)}}\left(\mathbf{C} \backslash \mathrm{I}_{\mathbf{C}}\right) \cup \cup_{i=1}^{t} \mathrm{~V}_{\overline{K(U)}}\left(\mathbf{P} \cup \mathbf{C} \cup\left\{\mathrm{I}_{C_{i}}\right\}\right)
$$

On the base of Theorem 1, there exists an algorithm, namely Wu's method, for computing a finite sequence of ascending chains $\mathbf{C}_{1}, \mathbf{C}_{2}, \ldots, \mathbf{C}_{m}(m \geq 1)$ in $K[U][X]$ such that

(1) $\mathbf{C}_{1}, \mathbf{C}_{2}, \ldots, \mathbf{C}_{m}$ is a finite sequence of characteristic sets in $K[U][X]$;

(2)If $m=1, \mathrm{~V}_{\overline{K(U)}}(\mathbf{P})=\emptyset$. Otherwise, suppose $\mathbb{S}=\left\{\mathbf{C}_{i} \mid 1 \leq i \leq m\right.$ and $\mathbf{C}_{i}$ is a noncontradictory ascending chain\}, then $\mathrm{V}_{\overline{K(U)}}(\mathbf{P})=\cup_{\mathbf{C} \in \mathbb{S}} \mathrm{V}_{\overline{K(U)}}\left(\mathbf{C} \backslash \mathrm{I}_{\mathbf{C}}\right)$.

The set of ascending chains $\left\{\mathbf{C}_{1}, \mathbf{C}_{2}, \ldots, \mathbf{C}_{m}\right\}$ above is said to be a $W u$ 's decomposition or characteristic set decomposition of $\mathbf{P}$ in $K[U][X]$.

For any triangular set $\mathbf{T}$ in $K[U][X]$, we denote $\#(X)-\#(\mathbf{T})$ by $d(\mathbf{T}, X)$.

Definition 1. Let $\mathbf{P}$ be a parametric system in $K[U][X]$ and the set $\left\{\mathbf{C}_{1}, \mathbf{C}_{2}, \ldots, \mathbf{C}_{m}\right\}$ of ascending chains be a Wu's decomposition of $\mathbf{P}$ in $K[U][X]$. If $d\left(\mathbf{C}_{i}, X\right)=0$ for every non-contradictory ascending chain $\mathbf{C}_{i}, \mathbf{P}$ is said to be a generic zero-dimensional system. Otherwise, $\mathbf{P}$ is said to be a generic positive-dimensional system.

Definition 2. Let $\mathbf{P}$ be a parametric system in $K[U][X]$ and $\mathbb{T H} \mathbb{H}=\left\{\left[\mathbf{T}_{1}, H_{1}\right], \ldots,\left[\mathbf{T}_{s}, H_{s}\right]\right\}$ be a set of regular systems in $K[U][X]$. THI is said to be a parametric regular system decomposition of $\mathbf{P}$ in $K[U][X]$, if $\mathrm{V}_{\overline{K(U)}}(\mathbf{P})=\cup_{i=1}^{s} \mathrm{~V} \overline{K(U)}\left(\mathbf{T}_{i} \backslash H_{i}\right)$.

\footnotetext{
${ }^{1}$ The definition of regular system is the same as that introduced in 4 and different from that proposed in 22 , see more details in 4 .
} 
Definition 3. 4 Let $[\mathbf{T}, H]$ be a regular system in $K[U][X]$ and $a \in \bar{K}^{d}$. If $\mathbf{T}(a)$ is a regular chain in $\bar{K}[X], \operatorname{rank}(\mathbf{T}(a))=\operatorname{rank}(\mathbf{T})$ and $\operatorname{res}(H(a), \mathbf{T}(a)) \neq 0$, then we say that the regular system $[\mathbf{T}, H]$ specializes well at $a$.

Definition 4. Let $\mathbf{P}$ be a parametric system in $K[U][X]$ and $\mathbb{T H}=\left\{\left[\mathbf{T}_{1}, H_{1}\right], \ldots,\left[\mathbf{T}_{s}, H_{s}\right]\right\}$ be a parametric regular system decomposition of $\mathbf{P}$ in $K[U][X]$. For any $a \in \bar{K}^{d}$, if $\mathrm{V}(\mathbf{P}(a))=$ $\left.\cup_{i=1}^{s} \mathrm{~V}\left(\mathbf{T}_{i}(a) \backslash H_{i}(a)\right)\right)$ and $\left[\mathbf{T}_{i}, H_{i}\right](1 \leq i \leq s)$ specializes well at a, then $\mathbb{T} \mathbb{H}$ is said to be stable at a.

Remark that the concept of stable generic regular (chain) decomposition is first introduced in 19 .

Definition 5. Let $\mathbb{T H}$ be a parametric regular system decomposition of a given parametric system $\mathbf{P}$ in $K[U][X]$. If there is an affine variety $\mathcal{V}$ in $\bar{K}^{d}$ with $\operatorname{dim}(\mathcal{V})<d$ such that $\mathbb{T} \mathbb{H}$ is stable at any $a \in \bar{K}^{d} \backslash \mathcal{V}$, then $\mathbb{T H}$ is said to be a generic regular system decomposition of $\mathbf{P}$ and $\mathcal{V}$ is said to be a regular-decomposition-unstable variety (RDU) of $\mathbf{P}$ w.r.t. $\mathbb{T H}$.

Definition 6. Let $\mathbf{T}$ be a zero-dimensional regular chain in $K[U][X]$ and $P \in K[U][X]$. Suppose $\mathbb{H}$ and $\mathbb{G}$ are two finite sets of zero-dimensional regular chains in $K[U][X]$. If

(1) $\mathrm{V}_{\overline{K(U)}}(\mathbf{T} \cup\{P\})=\cup_{\mathbf{H} \in \mathbb{H}} \mathrm{V}_{\overline{K(U)}}(\mathbf{H})$,

(2) $\mathrm{V}_{\overline{K(U)}}(\mathbf{T} \backslash P)=\cup_{\mathbf{G} \in \mathbb{G}} \mathrm{V}_{\overline{K(U)}}(\mathbf{G})$,

then $(\mathbb{H}, \mathbb{G})$ is said to be a weakly relatively simplicial decomposition of $\mathbf{T}$ w.r.t. $P$ in $K[U][X]$.

In [19], we gave an algorithm for computing weakly relatively simplicial decompositions. Here, we only present its specification but omit the details.

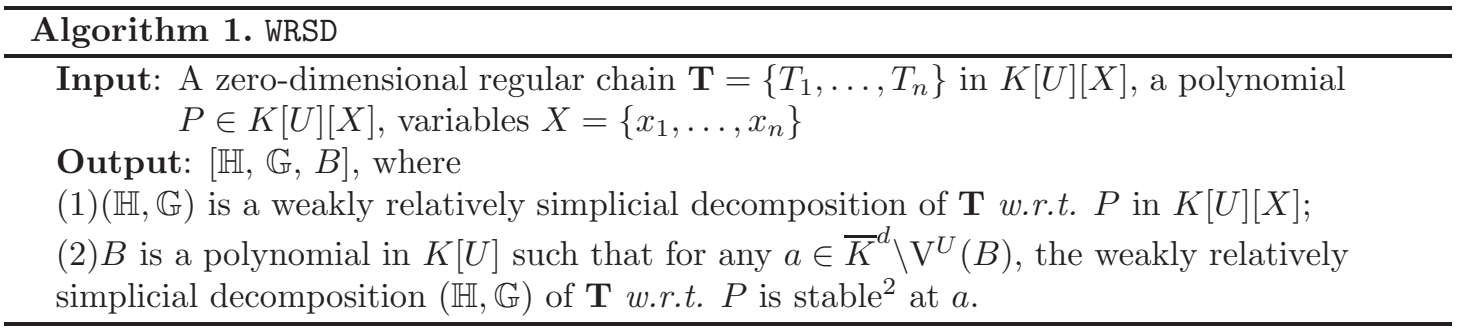

\section{Theory and Algorithm}

We first give some notations. Assume that $\mathrm{Alg}$ is a name of an algorithm and $p_{1}, \ldots, p_{t}$ is a sequence of inputs of this algorithm. If the output of $\operatorname{Alg}\left(p_{1}, \ldots, p_{t}\right)$ is a finite sequence $q_{1}, \ldots, q_{s}, q_{i}$ is denoted by $\operatorname{Alg}\left(p_{1}, \ldots, p_{t}\right)_{i}$ for any $i(1 \leq i \leq s)$ and also said to be the $i$ th output of $\operatorname{Alg}\left(p_{1}, \ldots, p_{t}\right)$. Given a finite set $S=\left\{s_{1}, \ldots, s_{t}\right\}$ and a map $\phi$ on $S, o p(S)$ denotes the finite sequence $s_{1}, \ldots, s_{t}$ and $\operatorname{map}(s \rightarrow \phi(s), S)$ denotes the set $\phi(S)=\{\phi(s) \mid s \in S\}$.

\subsection{Wu's Decomposition Under Specification}

The general idea of Wu's method is presented in Section 2. Some results on Wu's decomposition under specification are given in this section.

Definition 7. Let $\mathbf{P}_{1}$ be a parametric system in $K[U][X]$ and $\mathbb{S}=\left\{\mathbf{C}_{1}, \ldots, \mathbf{C}_{m}\right\}$ be a Wu's decomposition of $\mathbf{P}_{1}$ in $K[U][X]$. Suppose $\mathcal{L}=\left\{\mathbf{C}_{l, 1}, \mathbf{C}_{l, 2}, \ldots, \mathbf{C}_{l, k}\right\}$ is a subset of $\mathbb{S}$ satisfying that

(1) $\mathbf{C}_{l, 1}$ is a characteristic set of $\mathbf{P}_{1}$.

(2) If $k \geq 2, \mathbf{C}_{l, i}(2 \leq i \leq k)$ is a characteristic set of $\mathbf{P}_{i}=\mathbf{P}_{i-1} \cup \mathbf{C}_{l, i-1} \cup\left\{\mathrm{I}_{C_{l, i-1}}\right\}$ where $C_{l, i-1} \in \mathbf{C}_{l, i-1}$.

\footnotetext{
${ }^{2}$ Please see the definition of stable in [19].
} 
(3) If $k=1, \mathbf{C}_{l, 1}$ is a contradictory ascending chain. Otherwise, $\mathbf{C}_{l, i}(1 \leq i \leq k-1)$ is a non-contradictory ascending chain and $\mathbf{C}_{l, k}$ is a contradictory ascending chain.

Then $\mathcal{L}$ is said to be a line of $\mathbb{S}$ and $\mathbf{P}^{\mathcal{L}}=\left\{\mathbf{P}_{1}, \ldots, \mathbf{P}_{k}\right\}$ the corresponding systems.

Lemma 1. Let $\mathbf{P}_{1}$ be a parametric system in $K[U][X]$ and $\mathbb{S}=\left\{\mathbf{C}_{1}, \ldots, \mathbf{C}_{m}\right\}$ be a Wu's decomposition of $\mathbf{P}_{1}$ in $K[U][X]$. Let $\mathcal{L}=\left\{\mathbf{C}_{l, 1}, \ldots, \mathbf{C}_{l, k}\right\}$ be a line of $\mathbb{S}$ with corresponding systems $\left\{\mathbf{P}_{1}, \ldots, \mathbf{P}_{k}\right\}$. Then for any $a \in \bar{K}^{d} \backslash \mathrm{V}^{U}\left(\mathbf{C}_{l, k}\right)$, there exists a polynomial $p_{i} \in \mathbf{P}_{i}$ such that $p_{i}(a) \not \equiv 0$ for any $i(1 \leq i \leq k)$.

Proof. We prove it by induction on the number $\mathrm{k}$ of elements of $\mathcal{L}$. If $\mathrm{k}=1$, it means $\mathcal{L}$ contains only one element. Since $\mathbf{C}_{l, 1}$ is a contradictory ascending chain, we can assume that $\mathbf{C}_{l, 1}=\left\{C_{l, 1}\right\}$ where $C_{l, 1} \in K[U]$ and we know that $C_{l, 1} \in\left\langle\mathbf{P}_{1}\right\rangle$. Suppose $\mathbf{P}_{1}=\left\{f_{1,1}, \ldots, f_{1, t_{1}}\right\}$. Then $C_{l, 1}$ can be written as $C_{l, 1}=\sum_{j=1}^{t_{1}} h_{j} f_{1, j}$ where $h_{j} \in K[U][X]$ for any $j\left(1 \leq j \leq t_{1}\right)$. Thus $C_{l, 1}(a)=\sum_{j=1}^{t_{1}} h_{j}(a) f_{1, j}(a)$ holds. Since $C_{l, 1}(a) \not \equiv 0$, there must exist some $f_{1, e_{1}} \in \mathbf{P}_{1}$ such that $f_{1, e_{1}}(a) \not \equiv 0$. Let $p_{1}=f_{1, e_{1}}$ and we are done.

Now we assume that the conclusion holds when $k<N(N>1)$. Suppose $k=N$. $\mathcal{L}_{2}=\left\{\mathbf{C}_{l, 2}, \ldots, \mathbf{C}_{l, k}\right\} \subsetneq \mathcal{L}$ is a line of Wu's decomposition of $\mathbf{P}_{2}$. According to the induction hypothesis, for any $a \in \bar{K}^{d} \backslash \mathrm{V}^{U}\left(\mathbf{C}_{l, k}\right)$, there exists a polynomial $p_{i} \in \mathbf{P}_{i}$ such that $p_{i}(a) \not \equiv 0$ for any $i(2 \leq i \leq k)$. As is known to us, $\mathbf{P}_{2}=\mathbf{P}_{1} \cup \mathbf{C}_{l, 1} \cup\left\{\mathrm{I}_{C_{l, 1}}\right\}$, if $p_{2} \in \mathbf{P}_{1}$, let $p_{1}=p_{2}$ and the conclusion holds obviously. Otherwise, $p_{2} \in\left\{\mathrm{I}_{C_{l, 1}}\right\} \cup \mathbf{C}_{l, 1}$. If $p_{2} \in \mathbf{C}_{l, 1} \subset\left\langle\mathbf{P}_{1}\right\rangle$, supposing $\mathbf{P}_{1}=\left\{f_{1,1}, \ldots, f_{1, t_{1}}\right\}$, then $p_{2}$ can be written as $p_{2}=\sum_{j=1}^{t_{1}} h_{j} f_{1, j}$ where $h_{j} \in K[U][X]$ for any $j\left(1 \leq j \leq t_{1}\right)$. Thus $p_{2}(a)=\sum_{j=1}^{t_{1}} h_{j}(a) f_{1, j}(a)$ holds. Since $p_{2}(a) \not \equiv 0$, there must exist some $f_{1, e_{1}} \in \mathbf{P}_{1}$ such that $f_{1, e_{1}}(a) \not \equiv 0$. Let $p_{1}=f_{1, e_{1}}$ and we are done. If $p_{2}=\left\{\mathrm{I}_{C_{l, 1}}\right\}$, it implies $C_{l, 1}(a) \not \equiv 0$. Since $C_{l, 1}$ can be written as $C_{l, 1}=\sum_{j=1}^{t_{1}} g_{j} f_{1, j}$ where $g_{j} \in K[U][X]$ for any $j$ $\left(1 \leq j \leq t_{1}\right)$. Thus there must exist some $f_{1, e_{1}} \in \mathbf{P}_{1}$ such that $f_{1, e_{1}}(a) \not \equiv 0$. Let $p_{1}=f_{1, e_{1}}$ and the conclusion holds.

With Lemma 1, Corollary 1 1 holds obviously.

Corollary 1. Let $\mathbf{P}$ be a parametric system in $K[U][X]$ and $\mathbb{S}=\left\{\mathbf{C}_{1}, \ldots, \mathbf{C}_{m}\right\}$ be a Wu's decomposition of $\mathbf{P}$ in $K[U][X]$. Let $\mathcal{L}=\left\{\mathbf{C}_{l, 1}, \mathbf{C}_{l, 2}, \ldots, \mathbf{C}_{l, k}\right\}$ be a line of $\mathbb{S}$. If $\mathbf{P}=\{p\}$, containing only one polynomial in $K[U][X], p(a) \not \equiv 0$ for any $a \in \bar{K}^{d} \backslash \mathrm{V}^{U}\left(\mathbf{C}_{l, k}\right)$.

Lemma 2. Suppose $\mathbf{C}=\left\{C_{1}, \ldots, C_{t}\right\}$ is a non-contradictory ascending chain and a characteristic set of parametric system $\mathbf{P}$ in $K[U][X]$. For any $a \in \bar{K}^{d}, \mathrm{~V}(\mathbf{P}(a))=\mathrm{V}\left(\mathbf{C}(a) \backslash \mathrm{I}_{\mathbf{C}}(a)\right) \cup$ $\cup_{i=1}^{t} \mathrm{~V}\left(\left(\mathbf{P}(a) \cup \mathbf{C}(a) \cup\left\{\mathrm{I}_{C_{i}}(a)\right\}\right)\right)$.

Proof. For any $a \in \bar{K}^{d}$, if $\mathrm{I}_{\mathbf{C}}(a) \equiv 0$, the conclusion holds obviously since $\mathrm{I}_{\mathbf{C}}(a)=\prod_{i=1}^{t} \mathrm{I}_{C_{i}}(a)$. Now we prove the conclusion when $\mathrm{I}_{\mathbf{C}}(a) \not \equiv 0$. According to the definition of characteristic set, we know that $\mathbf{C} \subset\langle\mathbf{P}\rangle$ and for any $p \in \mathbf{P}, \mathrm{I}_{C_{1}}{ }^{k_{1}} \cdots \mathrm{I}_{C_{t}}{ }^{k_{t}} p=q_{1} C_{1}+\cdots+q_{t} C_{t}$ where $q_{i} \in K[U][X]$ for any $i(1 \leq i \leq t)$. Then $\mathbf{C}(a) \subset\langle\mathbf{P}(a)\rangle$ and $\mathrm{I}_{C_{1}}(a)^{k_{1}} \cdots \mathrm{I}_{C_{t}}(a)^{k_{t}} p(a)=$ $q_{1}(a) C_{1}(a)+\cdots+q_{t}(a) C_{t}(a)$. Therefore $\mathrm{V}\left(\mathbf{C}(a) \backslash \mathrm{I}_{\mathbf{C}}(a)\right) \subset \mathrm{V}(\mathbf{P}(a)) \subset \mathrm{V}(\mathbf{C}(a))$ and $\mathrm{V}(\mathbf{P}(a))=$ $\mathrm{V}\left(\mathbf{C}(a) \backslash \mathrm{I}_{\mathbf{C}}(a)\right) \cup \mathrm{V}\left(\mathbf{P}(a) \cup\left\{\mathrm{I}_{\mathbf{C}}(a)\right\}\right)$. Since $\mathbf{C}(a) \subset\langle\mathbf{P}(a)\rangle, \mathrm{V}\left(\mathbf{P}(a) \cup\left\{\mathrm{I}_{\mathbf{C}}(a)\right\}\right)=\mathrm{V}(\mathbf{P}(a) \cup \mathbf{C}(a) \cup$ $\left.\mathrm{I}_{\mathbf{C}}(a)\right)=\cup_{i=1}^{t} \mathrm{~V}\left(\left(\mathbf{P}(a) \cup \mathbf{C}(a) \cup\left\{\mathrm{I}_{C_{i}}(a)\right\}\right)\right)$. We are done.

According to Theorem 1 and Lemma 2 we can get Corollary 2 easily.

Corollary 2. Let $\mathbf{P}$ be a parametric system in $K[U][X]$ and $\left\{\mathbf{C}_{1}, \ldots, \mathbf{C}_{m}\right\}$ be a Wu's decomposition of $\mathbf{P}$ in $K[U][X]$. Suppose $\mathbb{S}=\left\{\mathbf{C}_{i} \mid 1 \leq i \leq m\right.$ and $\mathbf{C}_{i}$ is a non-contradictory ascending chain $\}$ and $\mathbb{C S}=\left\{\mathbf{C}_{i} \mid 1 \leq i \leq m\right.$ and $\mathbf{C}_{i}$ is a contradictory ascending chain $\}$. Then for any $a \in \bar{K}^{d} \backslash\left(\cup_{\mathbf{C S} \in \mathbb{C S}} \mathrm{V}^{U}(\mathbf{C S})\right), \mathrm{V}(\mathbf{P}(a))=\cup_{\mathbf{C} \in \mathbb{S}} \mathrm{V}\left(\mathbf{C}(a) \backslash \mathrm{I}_{\mathbf{C}}(a)\right)$.

\subsection{Converting To Regular Systems}

Let $\mathbf{T}$ be a triangular set in $K[U][X]$. We can compute a set of finite sequence of regular systems $\mathbb{T H}=\left\{\left[\mathbf{T}_{1}, H_{1}\right], \ldots,\left[\mathbf{T}_{s}, H_{s}\right]\right\}$ in $K[U][X]$ and a polynomial $B \in K[U]$ on the basis 
of Algorithm 1 such that $\mathrm{V}_{\overline{K(U)}}\left(\mathbf{T} \backslash \mathrm{I}_{\mathbf{T}}\right)=\cup_{i=1}^{s} \mathrm{~V}_{\overline{K(U)}}\left(\mathbf{T}_{i} \backslash H_{i}\right)$ and for any $a \in \bar{K}^{d} \backslash \mathrm{V}^{U}(B)$, THH specializes well at $a$ and $\mathrm{V}\left(\mathbf{T}(a) \backslash \mathrm{I}_{\mathbf{T}}(a)\right)=\cup_{i=1}^{s} \mathrm{~V}\left(\mathbf{T}_{i}(a) \backslash H_{i}(a)\right)$. The algorithm is presented as Algorithm 3, which plays a key role in Algorithm 4 proposed in the next section.

Algorithm 2 below was proposed in [19] for zero-dimensional case. We just give its specification here. Our focus in this paper is how to deal with the case where the triangular set is positive-dimensional. So, we need to convert a triangular set with $\operatorname{mvar}(\mathbf{T}) \subsetneq \mathbf{X}$ to a set of regular systems.

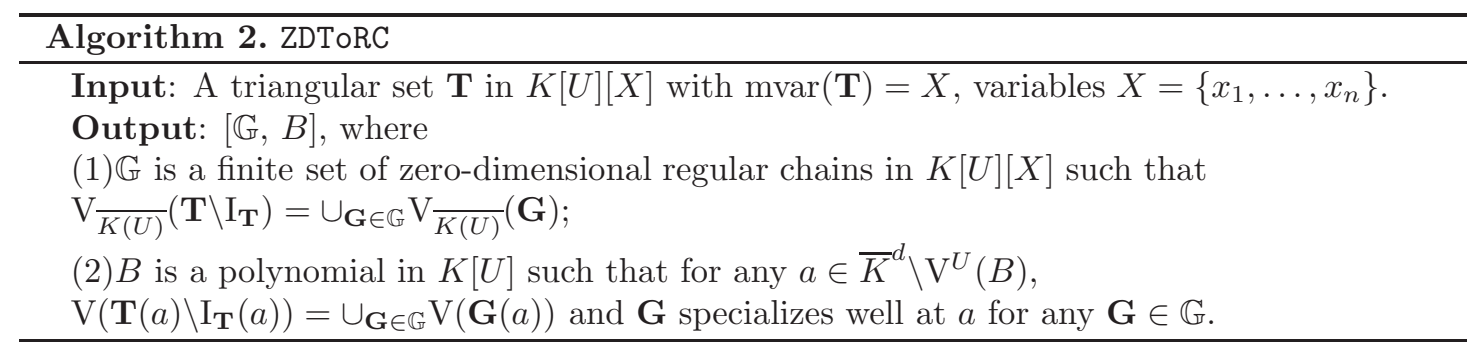

According to Algorithm 2, the following Proposition 2 is clear.

Proposition 2. Let $\mathbf{T}$ be a triangular set in $K[U][X]$ and $\operatorname{ZDToRC}(\mathbf{T}, \operatorname{mvar}(\mathbf{T}))=[\mathbb{G}, B]$. If $\mathbb{G} \neq \emptyset, \mathrm{I}_{\mathbf{T}}(a) \neq 0$ for $a \notin \mathrm{V}^{U}(B)$.

Now suppose $\mathbf{T}=\left\{T_{1}, \ldots, T_{l}\right\}$ is a triangular set in $K[U][X]$ and $\operatorname{mvar}(\mathbf{T}) \subsetneq X$ where variables $X=\left\{x_{1}, \ldots, x_{n}\right\}$ and parameters $U=\left\{u_{1}, \ldots, u_{d}\right\}$. It is interesting to show that we have two versions to interpret the relationship between $\mathbf{T}$ and the results computed by $\operatorname{ZDToRC}(\mathbf{T}, \operatorname{mvar}(\mathbf{T}))$. Assume that $\operatorname{ZDToRC}(\mathbf{T}, \operatorname{mvar}(\mathbf{T}))=[\mathbb{G}, B]$. Let $\mathcal{B}(\mathbf{T})=\operatorname{mvar}(\mathbf{T})$ and $\mathcal{F}(\mathbf{T})=X \backslash \operatorname{mvar}(\mathbf{T})$. On one hand, $\mathbf{T}$ can be regarded as a triangular set in $K[U, \mathcal{F}(\mathbf{T})][\mathcal{B}(\mathbf{T})]$. At this point, according to Algorithm 2, we know that

$(1) \mathrm{V}_{\overline{K(U, \mathcal{B}(\mathbf{T}))}}\left(\mathbf{T} \backslash \mathrm{I}_{\mathbf{T}}\right)=\cup_{\mathbf{G} \in \mathbb{G}} \mathrm{V}_{\overline{K(U, \mathcal{F}(\mathbf{T}))}}(\mathbf{G})$,

(2)for any $a \in \bar{K}^{d+n-l} \backslash \mathrm{V}^{U, \mathcal{F}(\mathbf{T})}(B), \mathrm{V}\left(\mathbf{T}(a) \backslash \mathrm{I}_{\mathbf{T}}(a)\right)=\cup_{\mathbf{G} \in \mathbb{G}} \mathrm{V}(\mathbf{G}(a))$ and $\mathbf{G}$ specializes well at $a$ for any $\mathbf{G} \in \mathbb{G}$ if $\mathbb{G} \neq \emptyset$.

On the other hand, $\mathbf{T}$ can also be regarded as a triangular set in $K[U][\mathcal{F}(\mathbf{T})][\mathcal{B}(\mathbf{T})]$. Let $\mathcal{K}=K(U), \mathcal{U}=\mathcal{F}(\mathbf{T})$ and $\mathcal{X}=\mathcal{B}(\mathbf{T})$. Then according to Algorithm 2 .

$(3) \mathrm{V}_{\overline{\mathcal{K}(\mathcal{U})}}\left(\mathbf{T} \backslash \mathrm{I}_{\mathbf{T}}\right)=\cup_{\mathbf{G} \in \mathbb{G}} \mathrm{V}_{\overline{\mathcal{K}(\mathcal{U})}}(\mathbf{G})$,

(4)for any $a \in \overline{\mathcal{K}}^{n-l} \backslash \mathrm{V}^{\mathcal{U}}(B), \mathrm{V}_{\overline{\mathcal{K}}}\left(\mathbf{T}(a) \backslash \mathrm{I}_{\mathbf{T}}(a)\right)=\cup_{\mathbf{G} \in \mathbb{G}} \mathrm{V}_{\overline{\mathcal{K}}}(\mathbf{G}(a))$ and $\mathbf{G}$ specializes well at $a$ for any $\mathbf{G} \in \mathbb{G}$ if $\mathbb{G} \neq \emptyset$.

Remark that the above statements (1) and (3) are exactly the same since $K(U, \mathcal{U})=\mathcal{K}(\mathcal{U})$. As discussed above, we have the following lemma by statements (2) and (4)

Lemma 3. Suppose $\mathbf{T}$ is a triangular set in $K[U][X]$ and $\operatorname{ZDToRC}(\mathbf{T}, \operatorname{mvar}(\mathbf{T}))=[\mathbb{G}, B]$. Then $\mathrm{V}_{\overline{K(U)}}\left(\mathbf{T} \backslash \mathbf{I}_{\mathbf{T}} \cdot B\right)=\cup_{\mathbf{G} \in \mathbb{G}} \mathrm{V}_{\overline{K(U)}}(\mathbf{G} \backslash B)$ and $\mathrm{V}\left(\mathbf{T}(a) \backslash \mathrm{I}_{\mathbf{T}}(a) \cdot B(a)\right)=\cup_{\mathbf{G} \in \mathbb{G}} \mathrm{V}(\mathbf{G}(a) \backslash B(a))$ for any $a \in \bar{K}^{d} \backslash \mathrm{V}^{U}(B)$.

\section{Theorem 2. Algorithm 3 terminates correctly.}

Proof. For a given triangular set $\mathbf{T}=\left\{T_{1}, \ldots, T_{l}\right\}$ in $K[U][X]$. Suppose ZDToRC $(\mathbf{T}, \operatorname{mvar}(\mathbf{T}))=$ $\left[\mathbb{G}_{0}, B_{0}\right]$.

Firstly, we prove Algorithm 3 terminates. If $B_{0} \in K[U]$, it terminates obviously. Otherwise, $B_{0} \in K[U][\mathcal{F}(\mathbf{T})] \subset K[U][X]$. Assume that $\left\{\mathbf{C}_{1}, \mathbf{C}_{2}, \ldots, \mathbf{C}_{m}\right\}$ is the Wu's decomposition of $\left\{B_{0}\right\}$ in $K[U][X]$ computed by Wu's method and $\mathbb{S}=\left\{\mathbf{C}_{i} \mid 1 \leq i \leq m\right.$ and $\mathbf{C}_{i}$ is a non-contradictory ascending chain $\}$. Obviously $m>1$ since $B_{0} \in K[U][\mathcal{F}(\mathbf{T})] \backslash K[U]$. For any $\mathbf{C}_{i} \in \mathbb{S}$, let $\mathbf{T}_{i}=$ $\mathbf{T} \cup \mathbf{C}_{i}$. Then we know that $\operatorname{mvar}(\mathbf{T}) \subsetneq \operatorname{mvar}\left(\mathbf{T}_{i}\right)$, which means $d\left(\mathbf{T}_{i}, X\right)<d(\mathbf{T}, X)$. Therefore, it is clearly that Algorithm 3 terminates within finite steps of recursion.

Now we prove the correctness by induction on the recursive depth $h$. If $h=1$, according to Algorithm [3 $B_{0} \in K[U]$ and the conclusion follows from Lemma 3. Assume that the conclusion holds for $h<N(N>1)$. When $h=N, B_{0} \in K[U][\mathcal{F}(\mathbf{T})] \backslash K[U]$. We can assume 


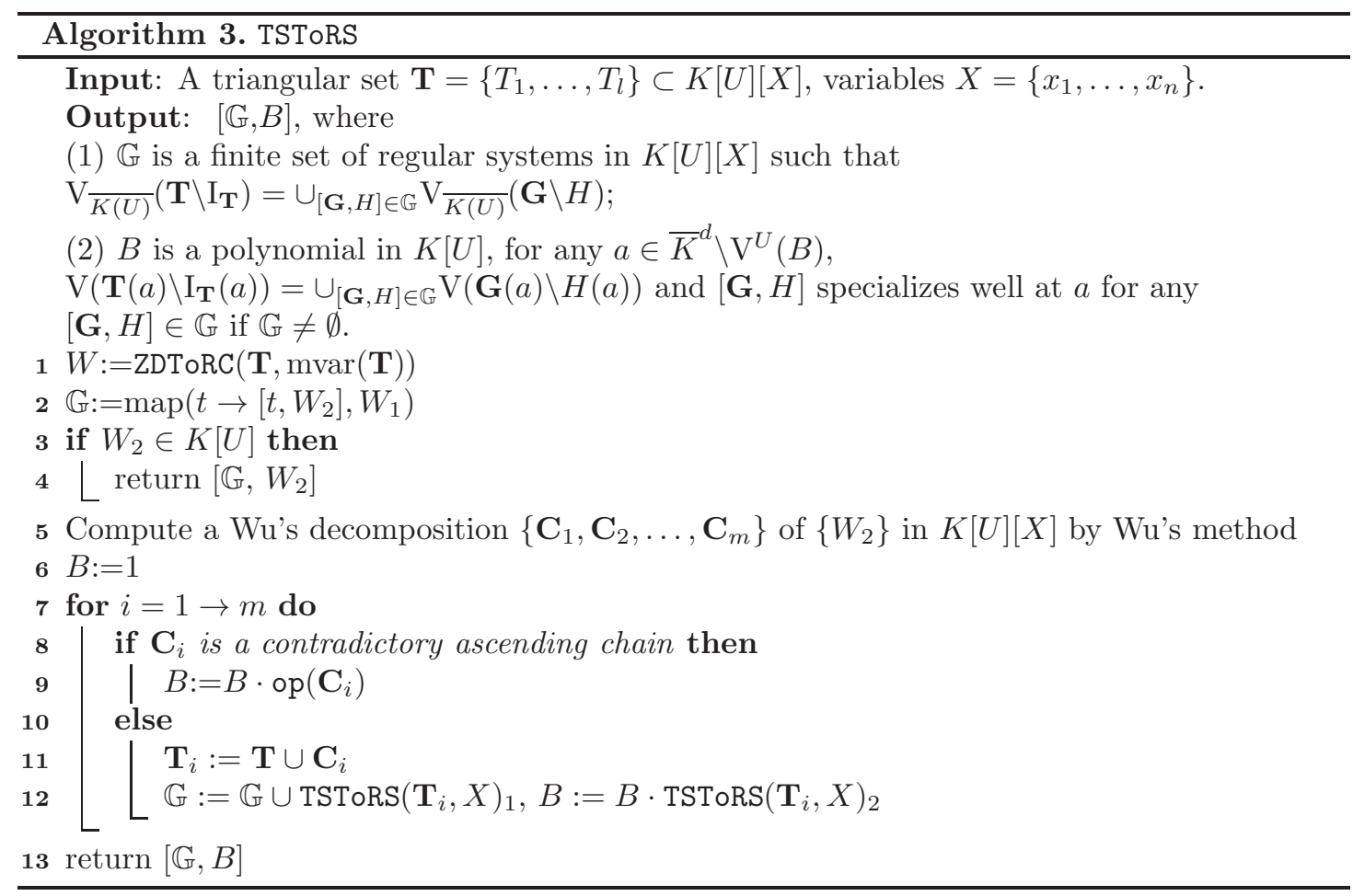

that $\left\{\mathbf{C}_{1}, \mathbf{C}_{2}, \ldots, \mathbf{C}_{m}\right\}$ is the Wu's decomposition of $\left\{B_{0}\right\}$ in $K[U][X]$ computed by Line 5 in Algorithm 3 Let $\mathcal{N}=\left\{i \mid 1 \leq i \leq m\right.$ and $\mathbf{C}_{i}$ is a non-contradictory ascending chain $\}$ and $\mathcal{C N}=\{1,2, \ldots, m\} \backslash \mathcal{N}$. For any $i \in \mathcal{N}$, let $\mathbf{T}_{i}=\mathbf{T} \cup \mathbf{C}_{i}$ and then $\mathbf{T}_{i}$ is a triangular set in $K[U][X]$. Suppose $\mathbf{C}_{j}=\left\{C_{j}\right\}(j \in \mathcal{C N})$, $\operatorname{TSToRS}\left(\mathbf{T}_{1}, \operatorname{mvar}\left(\mathbf{T}_{1}\right)\right)=\left[\mathbb{G}_{i}, B_{i}\right]$ for any $i(i \in \mathcal{N})$. Then according to Algorithm [3, $\mathbb{G}=\cup_{\mathbf{G} \in \mathbb{G}_{0}}\left\{\left[\mathbf{G}, B_{0}\right]\right\} \cup \cup_{i \in \mathcal{N}} \mathbb{G}_{i}$ and $B=\prod_{j \in \mathcal{C N}} C_{j} \cdot \prod_{i \in \mathcal{N}} B_{i}$. By Lemma 3. Wu's method and the induction hypothesis, we get

$$
\begin{aligned}
& \mathrm{V}_{\overline{K(U)}}\left(\mathbf{T} \backslash \mathrm{I}_{\mathbf{T}}\right) \\
& =\mathrm{V}_{\overline{K(U)}}\left(\mathbf{T} \backslash \mathrm{I}_{\mathbf{T}} \cdot B_{0}\right) \cup \mathrm{V}_{\overline{K(U)}}\left(\mathbf{T} \cup\left\{B_{0}\right\} \backslash \mathrm{I}_{\mathbf{T}}\right) \\
& =\cup_{\mathbf{G} \in \mathbb{G}_{0}} \mathrm{~V}_{\overline{K(U)}}\left(\mathbf{G} \backslash B_{0}\right) \cup\left(\mathrm{V}_{\overline{K(U)}}\left(\mathbf{T} \backslash \mathrm{I}_{\mathbf{T}}\right) \cap \mathrm{V}_{\overline{K(U)}}\left(B_{0}\right)\right) \\
& =\cup_{\mathbf{G} \in \mathbb{G}_{0}} \mathrm{~V}_{\overline{K(U)}}\left(\mathbf{G} \backslash B_{0}\right) \cup\left(\mathrm{V}_{\overline{K(U)}}\left(\mathbf{T} \backslash \mathrm{I}_{\mathbf{T}}\right) \cap \cup_{i \in \mathcal{N}} \mathrm{V}_{\overline{K(U)}}\left(\mathbf{C}_{i} \backslash \mathrm{I}_{\mathbf{C}_{i}}\right)\right) \\
& =\cup_{\mathbf{G} \in \mathbb{G}_{0}} \mathrm{~V}_{\overline{K(U)}}\left(\mathbf{G} \backslash B_{0}\right) \cup\left(\cup_{i \in \mathcal{N}} \mathrm{V}_{\overline{K(U)}}\left(\mathbf{T}_{i} \backslash \mathrm{I}_{\mathbf{T}_{i}}\right)\right) \\
& \left.=\cup_{\mathbf{G} \in \mathbb{G}_{0}} \mathrm{~V}_{\overline{K(U)}}\left(\mathbf{G} \backslash B_{0}\right) \cup\left(\cup_{i \in \mathcal{N}} \cup_{[\mathbf{G}, H] \in \mathbb{G}_{i}} \mathrm{~V}_{\overline{K(U)}}(\mathbf{G} \backslash H)\right)\right) .
\end{aligned}
$$

Therefore, the statement (1) in the specification of Algorithm 3 holds.

For any $a \in \bar{K}^{d} \backslash \mathrm{V}^{U}(B), C_{j}(a) \neq 0$ and $B_{i}(a) \neq 0$ for any $i \in \mathcal{N}$ and $j \in \mathcal{C N}$. Thus by Corollary 2, $\mathrm{V}\left(B_{0}(a)\right)=\cup_{i \in \mathcal{N}} \mathrm{V}\left(\mathbf{C}_{i}(a) \backslash \mathrm{I}_{\mathbf{C}_{i}}(a)\right)$. By Lemma 3 and the induction hypothesis, we get

$$
\begin{aligned}
& \mathrm{V}\left(\mathbf{T}(a) \backslash \mathrm{I}_{\mathbf{T}}(a)\right) \\
& =\mathrm{V}\left(\mathbf{T}(a) \backslash \mathrm{I}_{\mathbf{T}}(a) \cdot B_{0}(a)\right) \cup \mathrm{V}\left(\mathbf{T}(a) \cup\left\{B_{0}(a)\right\} \backslash \mathrm{I}_{\mathbf{T}}(a)\right) \\
& =\left(\cup_{\mathbf{G} \in \mathbb{G}_{0}} \mathrm{~V}\left(\mathbf{G}(a) \backslash B_{0}(a)\right)\right) \cup\left(\mathrm{V}\left(\mathbf{T}(a) \backslash \mathrm{I}_{\mathbf{T}}(a)\right) \cap \mathrm{V}\left(B_{0}(a)\right)\right) \\
& =\left(\cup_{\mathbf{G} \in \mathbb{G}_{0}} \mathrm{~V}\left(\mathbf{G}(a) \backslash B_{0}(a)\right)\right) \cup\left(\mathrm{V}\left(\mathbf{T}(a) \backslash \mathrm{I}_{\mathbf{T}}(a)\right) \cap \cup_{i \in \mathcal{N}} \mathrm{V}\left(\mathbf{C}_{i}(a) \backslash \mathrm{I}_{\mathbf{C}_{i}}(a)\right)\right) \\
& =\left(\cup_{\mathbf{G} \in \mathbb{G}_{0}} \mathrm{~V}\left(\mathbf{G}(a) \backslash B_{0}(a)\right)\right) \cup\left(\cup_{i \in \mathcal{N}} \mathrm{V}\left(\mathbf{T}_{i}(a) \backslash \mathrm{I}_{\mathbf{T}_{i}}(a)\right)\right) \\
& =\left(\cup_{\mathbf{G} \in \mathbb{G}_{0}} \mathrm{~V}\left(\mathbf{G}(a) \backslash B_{0}(a)\right)\right) \cup\left(\cup_{i \in \mathcal{N}} \cup_{[\mathbf{G}, H] \in \mathbb{G}_{i}} \mathrm{~V}(\mathbf{G}(a) \backslash H(a))\right) .
\end{aligned}
$$

In addition, by Lemma 1 and Corollary $1 B_{0}(a) \neq 0$. Thus $\left[\mathbf{G}, B_{0}\right]$ specializes well at $a$ for every $\mathbf{G} \in \mathbb{G}_{0}$ according to Algorithm 2 By the induction hypothesis, we know that $[\mathbf{G}, H]$ specializes well at $a$ for every $[\mathbf{G}, H] \in \mathbb{G}_{i}$ for any $i \in \mathcal{N}$. Therefore, the statement (2) in the specification of Algorithm 3 holds. 
Remark 1. By Algorithm [ for any regular system $\left[\mathbf{T}_{i}, H_{i}\right]$ in the first output of Algorithm 圆. $\operatorname{res}\left(\mathrm{I}_{\mathbf{T}_{i}}, \mathbf{T}_{i}\right)$ is a factor of $H_{i}$. If $B \in K[U]$ is the second output of Algorithm , by Corollary $\mathbf{1}$, we know that $\mathrm{V}_{\overline{K(U)}}\left(\operatorname{res}\left(\mathrm{I}_{\mathbf{T}_{i}}, \mathbf{T}_{i}\right)\right) \subset \mathrm{V}_{\overline{K(U)}}\left(H_{i}\right)$ and $\mathrm{V}^{U}\left(\operatorname{res}\left(\mathrm{I}_{\mathbf{T}_{i}}, \mathbf{T}_{i}\right)\right) \subset \mathrm{V}^{U}\left(H_{i}\right) \subset \mathrm{V}^{U}(B)$.

\subsection{Computing RDU}

We present the main result in this section. Algorithm 4 shows how to compute a generic regular system decomposition and the associated RDU of a given system simultaneously.

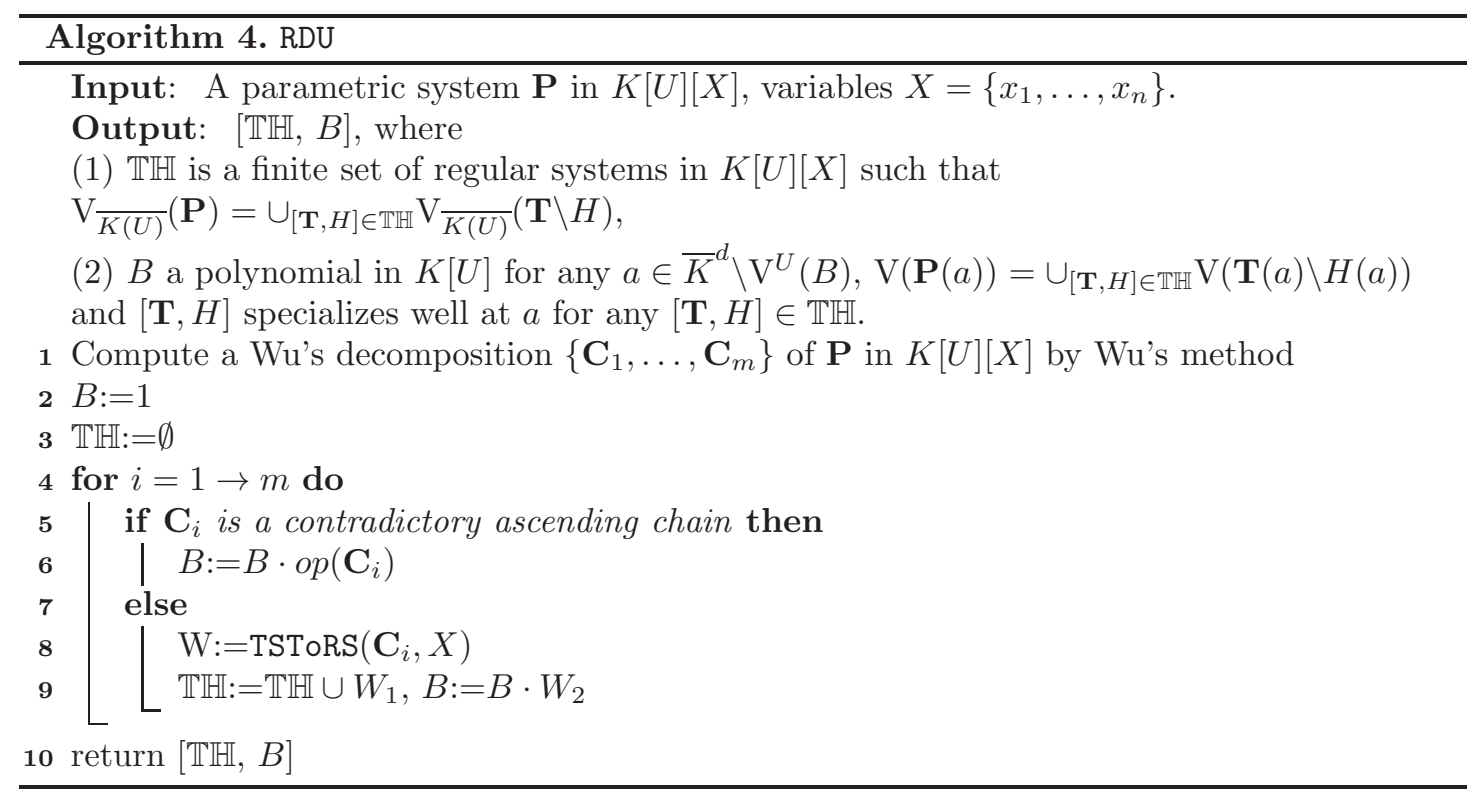

Theorem 3. Algorithm 田terminates correctly.

Proof. The termination follows from the termination of Algorithm 3. We only need to show the correctness. In fact, the statement (1) in the specification of Algorithm 4 follows from Wu's method and Algorithm 3 and the statement (2) follows from Corollary 2 and Algorithm 3

Corollary 3. Let $\mathbf{P}$ be a parametric polynomial system, $\operatorname{RDU}(\mathbf{P}, X)=[\mathbb{T H}, B]$. Then $\mathbb{T H}$ is stable at any $a \in \bar{K}^{d} \backslash \mathrm{V}^{U}(B)$.

The proof of Corollary 3 is similar to that of generic zero-dimensional case. For more details, please see [19].

\section{Examples and Implementation}

In this section, we show by examples how our algorithms work. In addition, we run some benchmarks and report comparison to other tools with similar function.

Example 1 is designed to illustrate how Algorithm 4 works.

Example 1. Consider the system

$$
\mathbf{P}=\left\{\begin{array}{l}
(u x+1) z^{3}+(v y+1) z^{2}+w x z+1 \\
u x+1
\end{array}\right.
$$

where $x, y$ and $z$ are variables $(x \prec y \prec z)$ and $u, v$ and $w$ are parameters.

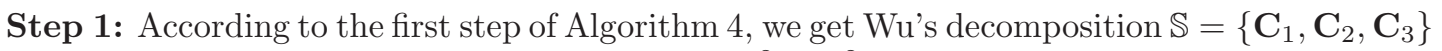
of $\mathbf{P}$ in $\mathbb{R}[u, v, w][x, y, z]$ where $\mathbf{C}_{1}=\left\{u x+1, u+u v y z^{2}+u z^{2}-w z\right\}, \mathbf{C}_{2}=\{u x+1, v y+1, u-w z\}$ and $\mathbf{C}_{3}=\left\{-v^{2} u^{5} w\right\}$. 
Step 2: Let $\mathbb{T H}=\emptyset$ and $B=1$.

Step 3: Because $\mathbf{C}_{1}$ and $\mathbf{C}_{2}$ are both non-contradictory ascending chains and $\mathbf{C}_{3}$ is a contradictory ascending chain, we need to execute $\left.\operatorname{TSToRS}\left(\mathbf{C}_{1},[x, y, z]\right\}\right)$ and $\operatorname{TSToRS}\left(\mathbf{C}_{2},[x, y, z]\right)$.

Step 3.1: According to TSToRS, we execute ZDToRC $\left(\mathbf{C}_{1}, \operatorname{mvar}\left(\mathbf{C}_{1}\right)\right)$ where $\operatorname{mvar}\left(\mathbf{C}_{1}\right)=$ $\{x, z\}$. It returns $W=\left[\left\{\left\{u x+1, u+u v y z^{2}+u z^{2} u-w z\right\}\right\}, u(v y+1)\right]$. Since $W_{2} \notin K[u, v, w]$, we execute wusolve $(u(v y+1))$ and it returns $\mathbf{C}_{11}=\{v y+1\}, \mathbf{C}_{12}=\{u\}$.

Step 3.2: Let $\mathbf{T}_{1}=\mathbf{T} \cup\{y v+1\}$. Now we need to execute $\operatorname{TSToRS}\left(\mathbf{T}_{1}\right)$. When we execute $\operatorname{ZDToRC}\left(\mathbf{T}_{1}, \operatorname{mvar}\left(\mathbf{T}_{1}\right)\right)$, it returns $[\emptyset, v u]$. Since $v u \in K[u, v, w]$, the output of $\left.\operatorname{TSToRS}\left(\mathbf{C}_{1},\{x, y, z\}\right\}\right)$ is $\left[\left\{\left[\left\{u x+1, u+u v y z^{2}+z^{2} \cdot u-w z\right\}, u(v y+1)\right]\right\}, u v w\right]$.

Step 3.3: Let $\mathbb{T H}:=\mathbb{T H} \cup\left\{\left[\left\{u x+1, u+u v y z^{2}+z^{2} \cdot u-w z\right\}, u(v y+1)\right]\right\}$ and $B=B \cdot u v w$.

Step 3.4: According to TSToRS, we execute $\operatorname{ZDToRC}\left(\mathbf{C}_{2}, \operatorname{mvar}\left(\mathbf{C}_{2}\right)\right)$ where $\operatorname{mvar}\left(\mathbf{C}_{2}\right)=$ $\{x, y, z\}$. It returns $W=[\{u x+1, v y+1, u-w z\},-u v w]$. Since $W_{2} \in K[u, v, w]$, the output of $\left.\operatorname{TSToRS}\left(\mathbf{C}_{2},[x, y, z]\right\}\right)$ is $[\{[\{u x+1, v y+1, u-w z\},-u v w]\}, u v w]$

Step 3.5: Let $\mathbb{T H}:=\mathbb{T} \mathbb{H} \cup\{[\{u x+1, v y+1, u-w z\},-u v w]\}$ and $B=B \cdot u v w$.

Step 3.6: Since $\mathbf{C}_{3}$ is a contradictory ascending chain, we execute $B:=B \cdot-v^{2} u^{5} w$.

Step 4: Finally, we get $B=u^{2} v^{2} w^{2}$ and $\mathbb{T}=\left\{\left[\left\{u x+1, u+u v y z^{2}+u z^{2}-w z\right\}, u(v y+\right.\right.$ $1)],[\{u x+1, v y+1, u-w z\},-u v w]\}$.

In the example, we factor the polynomials and let the polynomial be squarefree in some steps.

With the result above, we get a regular system decomposition of $\mathbf{P}$ in $\mathbb{R}[u, v, w][x, y, z]$ and a $\operatorname{RDU}$ variety $\mathcal{V}=\left\{(u, v, w) \in \mathbb{C}^{3} \mid u v w=0\right\}$. According to the specification of Algorithm 4 for any $a \in \mathbb{C}^{3} \backslash \mathcal{V}, \mathrm{V}(P(a))=\cup_{[\mathbf{T}, H] \in \mathbb{T H}} \mathrm{V}(\mathbf{T}(a) \backslash H(a))$ where $[\mathbf{T}(a), H(a)]$ is a regular system. With the definition of RDU variety, we get that $\mathcal{V}$ is the RDU variety of $\mathbf{P}$ w.r.t $\mathbb{T} H$.

Example 2 is provided by Changbo Chen, which is a good example to show how the orderings of variables affect the results and the efficiency of Algorithm 4 .

Example 2. Consider the parametric system

$$
\mathbf{P}=\left\{\begin{array}{l}
\left.d_{4} d_{3} r+r_{2}^{2}-d_{4} d_{3} r_{2}^{2}+d_{4}^{2} d_{3}^{2}-d_{4} d_{3}^{3}-d_{4}^{3} d_{3}+d_{4} d_{3}+Z-r\right) t^{4}+\left(-2 r_{2} d_{4} r+2 r_{2} d_{4}^{3}+\right. \\
\left.2 r_{2} d_{4} d_{3}^{2}-4 r_{2} d_{3} d_{4}^{2}+2 r_{2}^{3} d_{4}+2 r_{2} d_{4}\right) t^{3}-\left(2 r_{2}^{2}-2 r+4 d_{4}^{2} r_{2}^{2}+4 d_{4}^{2}+2 Z-2 d_{4}^{2} d_{3}^{2}\right) t^{2}+ \\
\left(-2 r_{2} d_{4} r+2 r_{2} d_{4} d_{3}^{2}+2 r_{2} d_{4}+2 r_{2} d_{4}^{3}+4 r_{2} d_{3} d_{4}^{2}+2 r_{2}^{3} d_{4}\right) t+r_{2}^{2}+d_{4}^{3} d_{3}-d_{4} d_{3} r+ \\
d_{4} d_{3} r_{2}^{2}+Z-r-d_{4} d_{3}+d_{4}^{2} d_{3}^{2}+d_{4} d_{3}^{3}
\end{array}\right.
$$

where $r, Z, t$ are variables and $r_{2}, d_{3}, d_{4}$ are parameters.

The 3 variables can be ordered in 6 different ways. We tried all these orders when calling Algorithm 4 for this example. We are interested in the RDU varieties output by the algorithm, so we only report the second output of the algorithm.

By calling $\operatorname{RDU}(\mathbf{P},[r, t, Z])$ and $\operatorname{RDU}(\mathbf{P},[t, r, Z])$, we get

$$
\begin{aligned}
& \operatorname{RDU}(\mathbf{P},[r, t, Z])_{2}=r_{2} d_{4} \\
& \operatorname{RDU}(\mathbf{P},[t, r, Z])_{2}=r_{2} d_{4}
\end{aligned}
$$

By calling $\operatorname{RDU}(\mathbf{P},[Z, r, t])$, we get

$$
\operatorname{RDU}(\mathbf{P},[Z, r, t])_{2}=\left(-d_{3}+d_{4} r_{2}^{2}+d_{4}\right) d_{4} r_{2}\left(d_{4} d_{3}-1\right)
$$

By calling $\operatorname{RDU}(\mathbf{P},[r, Z, t])$, we get

$$
\operatorname{RDU}(\mathbf{P},[r, Z, t])_{2}=r_{2} d_{4}\left(d_{3}-d_{4} r_{2}^{2}-d_{4}\right)
$$

By calling $\operatorname{RDU}(\mathbf{P},[t, Z, r])$, we get

$$
\operatorname{RDU}(\mathbf{P},[t, Z, r])_{2}=r_{2} d_{4}\left(d_{3}^{2}-3 r_{2}^{2}\right)\left(d_{4} d_{3}-1\right)
$$

$\operatorname{RDU}(\mathbf{P},[Z, t, r])_{2}$ is too huge to be listed here. It has 11 factors and contains 10838 terms (after expanding). 
Table 1. Timings (in second) of Example 0 under different orders of variables

\begin{tabular}{|c|c|c|c|c|c|c|}
\hline & {$[r, t, Z]$} & {$[t, r, Z]$} & {$[Z, r, t]$} & {$[r, Z, t]$} & {$[t, Z, r]$} & {$[Z, t, r]$} \\
\hline time & 0.016 & 0.015 & 0.016 & 0.046 & 0.016 & 3.931 \\
\hline
\end{tabular}

The timings for the above computation are shown in Table1. How to choose a suitable order in advance is an interesting topic for our future work.

Table 2. Comparing RDU and Triangularize

\begin{tabular}{|c|c|c|c|c|c|c|c|}
\hline number & system & $U$ & $X$ & wusolve & TSToRS & total & Triangularize \\
\hline 1. & Hereman-2 & 1 & 7 & 0.063 & 0. & 0.063 & 0.452 \\
\hline 2. & Hereman-8-8 & 3 & 5 & 0.281 & 0.015 & 0.296 & 0.188 \\
\hline 3. & Maclane & 3 & 7 & 0.093 & 0.078 & 0.171 & 0.156 \\
\hline 4. & MontesS7 & 1 & 3 & 0.967 & 0.015 & 0.982 & 0.063 \\
\hline 5. & MontesS11 & 3 & 3 & 0.016 & 0. & 0.016 & 0 \\
\hline 6. & MontesS12 & 2 & 6 & 0.109 & 0.078 & 0.187 & 0.093 \\
\hline 7. & MontesS13 & 3 & 2 & 0.016 & 0 & 0.016 & 0.031 \\
\hline 8. & MontesS14 & 1 & 4 & 0.031 & 0.016 & 0.047 & 0.141 \\
\hline 9. & MontesS15 & 4 & 8 & 0. & 0. & 0. & 0.016 \\
\hline 10. & MontesS16 & 3 & 12 & 0.016 & 0 . & 0.016 & 0.156 \\
\hline 11. & MontesS18 & 2 & 3 & 0.296 & 0.031 & 0.327 & 0.811 \\
\hline 12. & AlkashiSinus & 3 & 6 & 0. & 0. & 0 & 0.047 \\
\hline 13. & Bronstein & 2 & 2 & 0.016 & 0 & 0.016 & 0.062 \\
\hline 14. & Cheaters-homotopy-easy & 4 & 3 & 0.358 & 10.749 & $11.10^{7}$ & 0.047 \\
\hline 15. & Cheaters-homotopy-hard & 5 & 2 & 0. & 32.867 & 32.867 & 0.062 \\
\hline 16. & Gerdt & 3 & 4 & 0.015 & 0. & 0.015 & 0.016 \\
\hline 17. & Lanconelli & 7 & 4 & 0.047 & 0 . & 0.047 & 0 \\
\hline 18. & Lazard-ascm2001 & 3 & 4 & 0.811 & 0.047 & 0.858 & 0.39 \\
\hline 19. & Leykin-1 & 4 & 4 & 0.312 & 0. & 0.312 & 0.531 \\
\hline 20. & Neural & 1 & 3 & 0.047 & 0.016 & 0.063 & 0.093 \\
\hline 21. & Pavelle & 4 & 4 & 0.109 & 0.062 & 0.171 & 0.203 \\
\hline 22. & SY14 & 2 & 2 & 0.016 & 0. & 0.016 & 0 \\
\hline 23. & Wang93 & 2 & 3 & 0.031 & 0 & 0.031 & 0.046 \\
\hline 24. & zhou3 & 6 & 11 & 0.062 & 0.047 & 0.109 & 0.281 \\
\hline 25. & zhou4 & 4 & 7 & 0.016 & 0.016 & 0.032 & 0.093 \\
\hline 26. & $\mathrm{KdV}$ & 15 & 11 & 0.406 & 0. & 0.406 & 0.031 \\
\hline $2 \%$. & РЗР & 5 & 2 & 0 & 0.031 & 0.031 & 0.047 \\
\hline 28. & SBCD23 & 1 & 3 & 0.031 & 0. & 0.031 & 0.047 \\
\hline 29. & SBCD24 & 1 & 4 & 0.655 & 0.016 & 0.671 & 0.312 \\
\hline
\end{tabular}

We have implemented our algorithms with Maple 16. More specifically, Wu's method for computing parametric triangular decompositions introduced in Section 2 is implemented as a function wusolve and Algorithm 1 is implemented as a function WRSD. We ran many examples collected from other papers [15, 12, 4. At the same time, we compare the running time with Triangularize ${ }^{3}$ in RegularChains which can compute regular decompositions of given polynomial systems with parameters. Throughout this section, all the results are obtained in Maple 16 using an Intel(R) Core(TM) i5 processor $(3.20 \mathrm{GHz}$ CPU and 2.5 GB total memory) and Windows 7 (32 bit). The empirical data about timings is presented in Table 2.

In Table 2, the column marked $U$ and $X$ mean the number of parameters and variables, respectively. The column marked wusolve means the time used by wusolve at the first step in RDU (see Algorithm 4). The column marked TSToRS means the time used by TSToRS. The column marked Triangularize means the time used by Triangularize. Some data which shows 0 . means that the data is less than 0.001 and ignored by system.

From Table 2 we find that the cost of wusolve takes up a majority of the total time in most examples when solving practical problems as shown. Comparing with Triangularize, for some examples, our algorithm is faster than Triangularize. As for the example Cheaters-homotopyeasy and Cheaters-homotopy-hard, the time used by WRSD seems too much. This is not reasonable and optimization should be done in the future.

\footnotetext{
${ }^{3}$ Please find more details on Triangularize from the help document of Maple 16.
} 


\section{Conclusions}

The focus of this paper is how to decompose a parametric system into regular systems at "parameter level" and the property of this decomposition under specification. We provide an algorithm for computing GRDs of parametric systems and the related RDU varieties simultaneously no matter the systems are generic zero-dimensional or positive-dimensional, which is a generalization of our earlier work in [19] for generic zero-dimensional case. Then any parametric system in $K[U][X]$ can be decomposed into finitely many regular systems and the decomposition is stable at any parameter value in the complement of the associated RDU variety of the parameter space. That is to say, once we obtain such decomposition, all the solutions of the original system are expressed by some regular systems, except for some possible solutions over the parameter values on the associate RDU variety.

Note that, using Algorithm 4 of generic regular decomposition, we may get a complete decomposition of a given parametric system $\mathbf{P}$ step by step. A rough procedure is as follows. Firstly, for certain variables $X$ and parameters $U$, we call $\operatorname{RDU}(\mathbf{P}, X)$ and get a set of regular systems and a parametric polynomial $B \in K[U]$. Then, let $U_{1}=U \backslash\{u\}$ and $X_{1}=X \cup\{u\}$ where $u \in U$ and we call $\operatorname{RDU}\left(\mathbf{P} \cup\{B\}, X_{1}\right)$ to get a set of regular systems and a parametric polynomial $B_{1} \in K\left[U_{1}\right]$. Continuing this iteration, until $U_{i}=\emptyset$ and $B_{i} \in K$ for some $i$, we can get a complete decomposition finally. This is what we called hierarchical strategy in Section 1. What can we benefit from this strategy? According to this method, we can stop at any step of the iteration especially when the computation is hard to be finished. Then we get a generic regular decomposition and a set of parametric polynomials which can determinate a RDU variety (low dimensional variety). For some huge problems, limited by the computational capacity of micro computer, we can get a partial solution, which is useful if one cannot get any information by other complete methods. Of course, the procedure should be described clearly and proved to

be correct. That is one of our future work.

\section{Acknowledgements}

The work is partly supported by the National Natural Science Foundation of China (Grant No.11271034, No.11290141) and the project SYSKF1207 from SKLCS, IOS, the Chinese Academy of Sciences. Thank Changbo Chen and Yao Sun for providing a great deal of test-systems.

\section{References}

[1] P. Aubry, D. Lazard, M. Moreno Maza, On the Theories of Triangular Sets. J. Symb. Comp., 28: 105-124, 1999.

[2] C.B. Chen, J. Davenport, J.P. May, M. Moreno Maza, B.C. Xia, R. Xiao, Triangular Decomposition of Semi-Algebraic Systems. Proc. ISSAC 2010, 187-194, ACM Press, 2010.

[3] C.B. Chen, J. Davenport, M. Moreno Maza, B.C. Xia, R. Xiao, Computing with SemiAlgebraic Sets Represented by Triangular Decomposition. Proc. ISSAC 2011, 75-82, ACM Press, 2011.

[4] C.B. Chen, O. Golubitsky, F. Lemaire, M. Moreno Maza, W. Pan, Comprehensive Triangular Decomposition. Proc. CASC 2007, LNCS 4770, 73-101, 2007.

[5] D. Cox, J. Little, D. O'Shea, Using Algebraic Geometry, Springer, New York, 1998

[6] D. Cox, J. Little, D. O’Shea, Ideals, Varieties and Algorithms, Springer, New York, 2007

[7] X.S. Gao, S.C. Chou, Solving Parametric Algebraic Systems. Proc. ISSAC 1992, 335-341, ACM Press, 1992.

[8] C.B. Chen, M. Moreno Maza, Algorithms for Computing Triangular Decomposition of Polynomial Systems, J. Symb. Comp., 47: 610-642, 2012 
[9] S.C. Chou, Mechanical Geometry Theorem Proving. D. Reidel Publishing Company, 1987.

[10] X.S. Gao, X R. Hou, J.L. Tang, H.F. Chen, Complete Solution Classification for the Perspective-Three-Point Problem. IEEE Tran. on PAMI, 930-943, 25(8), 2003.

[11] M. Kalkbrener, A Generalized Euclidean Algorithm for Computing for Computing Triangular Representationa of Algebraic Varieties. J. Symb. Comput. , 15, 143-167, 1993.

[12] D. Kapur, Y. Sun, D.K. Wang, A New Algorithm for Computing Comprehensive Gröbner Systems. Proc. ISSAC 2010, 25-28, ACM Press, 2010.

[13] M. M. Maza, On Triangular Decompositions of Algebraic Varieties. Technical Report TR 4/99, NAG Ltd, Oxford, UK, 1999. Presented at the MEGA-2000 Conference, Bath, England.

[14] B. Mishra, Algorithmic Algebra. Springer-Verlag, New York, 1993.

[15] A. Montes, T. Recio, Automatic Discovery of Geometry Theorems Using Minimal Canonical Comprehensive Gröbner Systems. ADG 2006, LNAI 4869, 113-138, 2007.

[16] K. Nabeshima, A Speed-Up of the Algorithm for Computing Comprehensive Gröbner Systems. Proc. ISSAC200\%, 299-306, 2007.

[17] A. Suzuki, Y. Sato, An Alternative Approach to Comprehensive Gröbner Bases. Proc. ISSAC2002, 255-261, 2002.

[18] A. Suzuki, Y. Sato, A Simple Algorithm to Compute Comprehensive Gröbner Bases. Proc. ISSAC2006, 326-331, ACM Press, 2006.

[19] X.X. Tang, Z.H. Chen, B.C. Xia, Generic Regular Decompositions for Generic Zero-Dimensional Systems, accepted by Science China: Information Sciences, http://arxiv.org/abs/1208.6112, 2012

[20] D.K. Wang, Zero Decomposition Algorithms for System of Polynomial Equations. Computer Mathematics, Proc. ASCM 2000, 67-70, 2000.

[21] A. Montes, T. Recio, Automatic Discovery of Geometry Theorems Using Minimal Canonical Comprehensive Gröbner Systems. In: Botana F., Recio T., eds. ADG 2006, LNAI 4869. Berlin Heidelberg: Springer-Verlag Berlin Heidelberg, 2007. 113-138

[22] D.M. Wang, Computing Triangular Systems and Regular Systems. J. Symb. Comput., 30: $221-236,2000$.

[23] D.M. Wang, Elimination methods. Springer, New York, 2001.

[24] D.M. Wang, Characteristic Sets and Zero Struct of Polynomial Sets. Research Institure for Symbolic Computation

[25] D.M. Wang, Elimination Practice: Software Tools and Applications. Imperial College Press, London, 2004.

[26] V. Weispfenning, Comprehensive Gröbner bases. J. Symb. Comp. , 14: 1-29, 1992.

[27] W.-t. Wu, Basic Principles of Mechanical Theorem Proving in Elementary Geometries. J. Syst. Sci. Math. Sci. , 4: 207-235, 1984.

[28] B.C. Xia, DISCOVERER: A Tool for Solving Semi-Algebraic Systems. ACM Commun. Comput. Algebra. , 41(3): 102-103, 2007.

[29] L. Yang, X.R. Hou, B.C. Xia, A Complete Algorithm for Automated Discovering of a Class of Inequality-Type Theorems. Science in China, Series F, 44(6):33-49, 2001.

[30] L. Yang, B C. Xia, Real Solution Classifications of a Class of Parameteric Semi-Algebraic Systems. In Algorithmic Algebra and Logic-Proc. the A3L2005, 281-289, Herstellung and Verlag, Norderstedt, 2005. 
[31] L. Yang, B.C. Xia, Automated Proving and Discovering Inequalities (in Chinese). Science Press, Beijing, 2008.

[32] L. Yang, J.Z. Zhang, Searching Dependency between Algebraic Equations: an Algorithm Applied to Automated Reasoning. Technical Report ICTP/91/6, International Center for Theoretical Physics, 1-12, 1991.

[33] L. Yang, J.Z. Zhang, Searching Dependency between Algebraic Equations: an Algorithm Applied to Automated Reasoning. Artificial intelligence in Mathematics, 147-156, Oxford University Press, 1994.

[34] L. Yang, J.Z. Zhang, X.R. Hou, A Criterion of Dependency between Algebraic Equations and its Applications. Proc. International Workshop on Mathematics Mechanization'1992 (Wu W-T, Cheng M-D eds), 110-134, International Academic Publishers, Beijing, 1992.

[35] L. Yang, J.Z. Zhang, X.R. Hou, Non-Linear Algebraic Formulae and Theorem Automated Proving, Shanghai Education Technology Publishers, Beijing, 1992. 